MRS Advances (C) 2017 Materials Research Society

DOI: $10.1557 /$ adv.2017.8

\title{
Rheological Characterization of Alginate Based Hydrogels for Tissue Engineering
}

\author{
Pengfei Duan* ${ }^{1,2}$, Nehir Kandemir* ${ }^{1}$, Jiajun Wang ${ }^{1}$, Jinju Chen ${ }^{1 \dagger}$ \\ ${ }^{1}$ School of Mechanical and Systems Engineering, Newcastle University, Newcastle upon Tyne, \\ UK \\ ${ }^{2}$ School of Chemical Engineering and Advanced Materials, Newcastle University, Newcastle \\ upon Tyne, UK \\ *These two authors contribute equally to this manuscript. \\ †Corresponding author: E-mail: Jinju.chen@ncl.ac.uk
}

\begin{abstract}
Hydrogels have been widely used in many applications from tissue engineering to drug delivery systems. For both tissue engineering and drug delivery, the mechanical properties are important because they would affect cell-materials interactions and injectability of drugs encapsulated in hydrogel carriers. Therefore, it is important to study the mechanical properties of these hydrogels, particularly at physiological temperature $\left(37^{\circ} \mathrm{C}\right)$. This study adopted strain sweep and frequency sweep rotational rheological tests to investigate the rheological characteristics of various tissue engineering relevant hydrogels with different concentrations at $37^{\circ} \mathrm{C}$. These hydrogels include alginate, RGD-alginate, and copolymerized collagen/alginate/fibrin. It has revealed that the addition of RGD has negligible effect on the elastic modulus and viscosity of alginate. Alginate gels have demonstrated shear thinning behavior which indicates that they are suitable candidates as carriers for cells or drug delivery. The addition of collagen and fibrin would reinforce the mechanical properties of alginate which makes it a strong scaffold material.
\end{abstract}

\section{INTRODUCTION}

Hydrogels are water-swollen, crosslinked polymeric structures with covalent bonds from the reaction of one or more co-monomers, physical crosslinks from chain entanglements, and hydrogen bonds and van der Waals interactions between chains or crystallites combining macromolecular chains [1]. Among various hydrogels, alginate and collagen have been widely used for tissue engineering [2,3]. Obtained from brown algae, alginate is a well-known biomaterial widely used in drug delivery applications and in tissue engineering because of its biocompatibility, low toxicity, relative low cost and, simple gelation with divalent cations $[4,5$, 6]. To improve the capability of tissue regeneration, alginate gel is often covalently modified with peptides containing the amino acid sequence of arginine-glycine-aspartic acid (Arg-GlyAsp, known as RGD) to promote cell adhesion [7]. These modified alginate gels have been demonstrated to sustain adhesion, proliferation, and expression of differentiated phenotype of skeletal muscle cells [2]. However, it remains elusive if RGD modification may affect the mechanical properties of alginate. Collagen is the most widely used tissue-derived polymer which constitutes a main component of extracellular matrices of mammalian tissues including tendons and ligaments, bones and cartilage, skin and arteries [8]. However, physically formed collagen gels are lack of mechanical strength. Therefore, it is proposed to chemically cross-link the collagen using alginate and fibrinogen [2]. Fibrinogen, produced from blood, forms fibrin gels by an enzymatic polymerization at room temperature in the presence of thrombin. Fibrin 
gels are also used in tissue engineering applications, especially for cell migration and wound healing but their mechanical properties are limited [2]. To overcome the disadvantage of relatively weak mechanical properties for both collagen and fibrin gels, it has been recently proposed to copolymerize collagen, fibrin with alginate to produce more suitable scaffolds [9].

Mechanical properties of hydrogel scaffolds can affect cell and materials interactions and the subsequent tissue regeneration [10]. Therefore, it is essential to characterize the mechanical properties of hydrogel scaffolds. However, there is limited information for the mechanical properties of RGD modified alginate hydrogels and collagen/alginate/fibrin hydrogels. Hence, the objective of this study is to investigate the rheological properties of these key hydrogels by oscillatory rheological tests.

\section{EXPERIMENTAL DETAILS}

Alginate powder (Type I, low EEO, A6013), alginic acid sodium salt, $\mathrm{HEPES}, \mathrm{CaCl}_{2}$ and $\mathrm{NaCl}, \mathrm{NaOH}$, Type I collagen derived from calf skin (Sigma, C9791), fibrinogen from bovine plasma (Sigma, F8630), thrombin from bovine plasma (Sigma,T4648), acetic acid (Sigma Aldrich, A6283), and Dulbecco's phosphate-buffered saline (DPBS) used in this study were purchased from Sigma-Aldrich Co.

\section{$\underline{\text { Alginate and RGD-Alginate Hydrogels Fabrication }}$}

Alginate and RGD-alginate gel were prepared by $\mathrm{CaCl}_{2}$ gelation. Alginate solution was prepared by dissolving $0.5 \%, 1 \%, 2 \%, 3 \%, 4 \%$ and $5 \%$ of sodium alginate powder in $0.9 \% \mathrm{NaCl}$, respectively. $\mathrm{CaCl}_{2}$ solution was prepared by dissolving $0.1 \mathrm{M} \mathrm{CaCl}_{2}$ in water with $2 \% \mathrm{HEPES}$ $(\mathrm{v} / \mathrm{v})$ powder at $\mathrm{pH}$ 7-7.5. A filter paper soaked in $\mathrm{CaCl}_{2}$ was placed under the mold, then the mold was filled with alginate solution and another filter paper soaked in $\mathrm{CaCl}_{2}$ was placed on top. The mold with the secured filter papers was immersed in $\mathrm{CaCl}_{2}$ solution and incubated until total gelation.

\section{Collagen/Alginate/Fibrin Copolymerized Hydrogel Fabrication}

$5 \% \mathrm{w} / \mathrm{v}$ sodium alginate solution and $10 \% \mathrm{w} / \mathrm{v}$ fibrinogen solution were prepared by dissolving the corresponding powder in DPBS. $0.5 \%, 1 \%, 2.5 \% \mathrm{w} / \mathrm{v}$ collagen solutions were prepared by dissolving collagen type I powders, with $2 \% \mathrm{v} / \mathrm{v}$ acetic acid. For collagen concentrations of $0.5 \%, 1 \%$ and $2.5 \%$, a final solution was prepared by mixing the corresponding concentration of collagen with fibrinogen and alginate solutions at a ratio of $2: 1: 1$. Five samples for each collagen concentration were prepared from the final solution with a size of $20 \mathrm{~mm}$ diameter and $2 \mathrm{~mm}$ thickness. The mold with the samples was kept in the incubator at $37^{\circ} \mathrm{C}$ for 40 minutes to allow sol-gel transition. The samples were then immersed for one minute in $10 \%$ $\mathrm{w} / \mathrm{v} \mathrm{CaCl}$ to crosslink alginate and then 15 minutes in $1 \mathrm{U} / \mathrm{ml}$ Thrombin solution to polymerize fibrinogen and obtain fibrin. The samples were stored at $4^{\circ} \mathrm{C}$ in plastic petri dishes.

\section{Mechanical Testing}

Rheological characterization of hydrogels was carried out at $37^{\circ} \mathrm{C}$ using a Malvern Kinexus Pro+ rotational rheometer (Worcestershire, UK). This rheometer is equipped with a solvent trap 
system to prevent dehydration of the hydrogels during the tests. A $20 \mathrm{~mm}$ diameter serrated parallel plates were used to avoid slippage. To identify the linear viscoelastic region (LVR), oscillatory strain sweep tests were applied between the strain values of $0.01 \%$ and $10 \%$.

Frequency sweep tests were then applied between $0.1 \mathrm{~Hz}$ and $10 \mathrm{~Hz}$ at a strain value within the LVR $\left(0.1 \%\right.$ in this study). For shear rate sweep tests, shear rate value varied between $0.1 \mathrm{~s}^{-1}$ and $100 \mathrm{~s}^{-1}$ to examine the possible shear thinning behavior. For each test, three replicates were made. The student t-test was used for statistical analysis.

\section{DISCUSSION}

\section{Alginate and RGD Modified Alginate Gels}

Figure 1 displays how shear storage moduli $\left(\mathrm{G}^{\prime}\right)$ and shear loss moduli $\left(\mathrm{G}^{\prime \prime}\right)$ change with frequency for various hydrogels. It can be seen that $G^{\prime}$ and $G^{\prime \prime}$ values are almost independent of the frequency within the range investigated, which demonstrated solid-like mechanical response. The values of $G^{\prime}$ are greater than $G^{\prime \prime}$ for all the gels, which suggests elastic properties dominate. Statistical analysis has revealed that there is significant difference for $0.5 \%$ and $1 \%$ alginate gels and their RGD modified counterparts in terms of $G^{\prime}$ and $G^{\prime \prime}$ values $(p \ll 0.05)$. But the change in $G^{\prime}$ value after RGD modification is very small. The $G^{\prime} / G^{\prime \prime}$ ratios remain almost the same after RGD modification. This suggests that the RGD modification has little effect on the mechanical properties of the alginate gels.

Alginate gels with concentration of 2-5\% have similar $\mathrm{G}^{\prime}(21.1-33.2 \mathrm{kPa})$ and $\mathrm{G}^{\prime \prime}(3.4-8.8$ $\mathrm{kPa}$ ) values. They are considerably stiffer than $1 \%$ and $0.5 \%$ alginate gels. The storage modulus $\mathrm{G}^{\prime}$ of $0.5 \%$ alginate obtained here agrees with what was reported in other similar work (i.e. $600-$ $1000 \mathrm{~Pa}$ at $1 \mathrm{~Hz}[11])$.
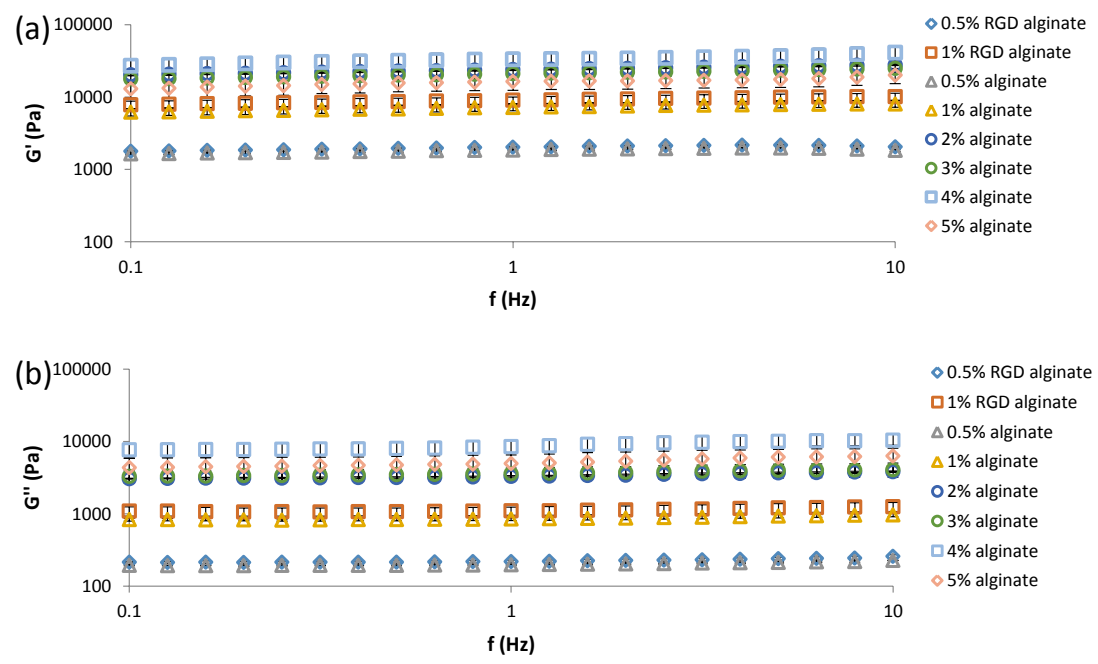

Figure 1. (a) Elastic modulus and (b) viscous modulus of alginate and RGD alginate gels 
Figure 2 shows how the viscosity of alginate gels changes with frequency and shear rate. The viscosity decreases with the increase of frequency and shear rate. The way how the viscosity $\eta^{*}$ changes is the same among all these hydrogels. This trend of viscosity may result from the change of the hindrance and friction among polymer chains [3]. For all these alginate based hydrogels, the viscosity significantly decreases with shear rate, which demonstrates shear thinning behavior. This suggests that alginate gels can be injected by applying high shear rate during injection and these types of gels can self-heal shortly after removing shear stress. Therefore, these gels can be used as carrier for biological molecules and cells during the injection.
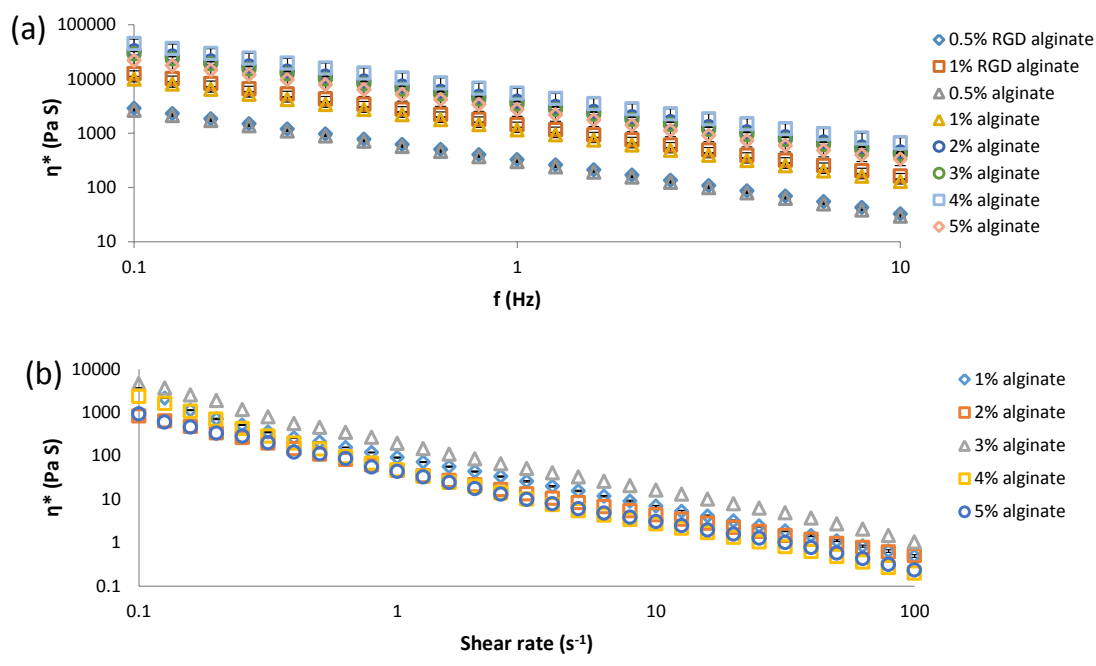

Figure 2. Viscosity of alginate gels as a function of (a) frequency and (b) shear rate

\section{Collagen/Alginate/Fibrin Gels}

Figure 3 displays how the elastic moduli, viscous moduli and viscosity change with frequency for various collagen/alginate/fibrin copolymerized hydrogels. The values of $\mathrm{G}^{\prime}, \mathrm{G}^{\prime \prime}$ and $\eta^{*}$ increase with collagen concentration. The values of $G^{\prime}$ and $G^{\prime \prime}$ slightly increase with the frequency. The $\mathrm{G}^{\prime} / \mathrm{G}^{\prime \prime}$ ratios are $5.40,5.94$ and 7.22 for $0.5 \%, 1 \%$ and $2.5 \%$ gels, respectively, which is elastic properties dominate. The frequency dependent $\mathrm{G}^{\prime}$ of hydrogels with $2.5 \%$ collagen is 1.93 times stiffer than hydrogels with $1 \%$ collagen, and 3.70 times stiffer than hydrogels with $0.5 \%$ collagen. There is significant difference for these collagen/alginate/fibrin gels in terms of $G^{\prime}$ and $G^{\prime \prime}(p \ll 0.05)$. Similar to the alginate gels, the viscosity $\eta^{*}$ of these copolymerized gels also decreases with frequency. In contrast to alginate gels (see Figure 1), the frequency dependent phenomena of collagen/alginate/fibrin gels demonstrated more liquid-like behavior. Similar frequency dependency of $\mathrm{G}^{\prime}$ and $\mathrm{G}^{\prime \prime}$ of type I collagen gel has also been reported in [12] and [13], in which case $\mathrm{G}^{\prime}$ and $\mathrm{G}^{\prime \prime}$ (in the order of $10 \mathrm{~Pa}$ ) also gently increase 
with frequency. This may suggest that additive alginate and fibrin can reinforce the strength of collagen gel, and the strength of this composite gel will be lower than that for alginate gel only. Similar to alginate gels, the viscosity $\eta^{*}$ of the copolymerized gels significantly decrease with frequency.
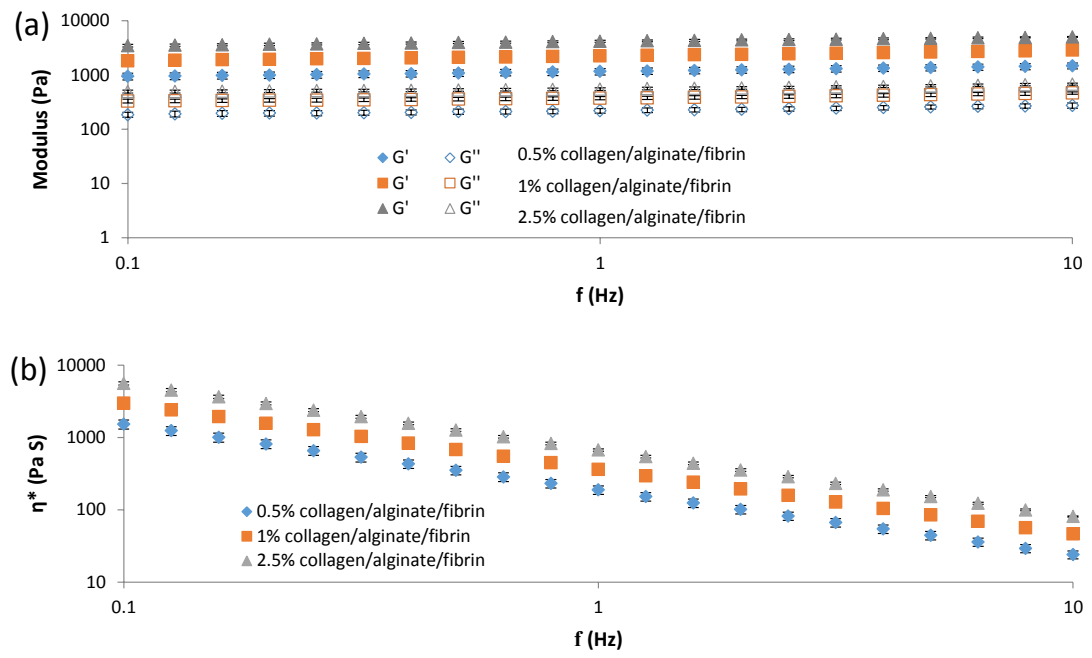

Figure 3. (a) Elastic and viscous modulus and (b) viscosity values of collagen/alginate/fibrin gels

\section{CONCLUSIONS}

All the alginate based hydrogels tested here have exhibited shear thinning behavior. It is found that the addition of RGD to alginate has very small effect on mechanical properties of the alginate gels.

For the copolymerized collagen/alginate/fibrin hydrogels, the values of $\mathrm{G}^{\prime}$ and $\mathrm{G}^{\prime \prime}$ are dependent on collagen concentration. These copolymerized gels are stiffer than their individual monomer gels. In addition, these collagen/alginate/fibrin copolymerized gels become frequency dependent in terms of both elastic modulus and viscous modulus even at linear viscoelastic region which may be correlated to the frequency-dependent diffusion in the network and local structure re-arrangement. This suggests that copolymerization does not only reinforce the mechanical strength of collagen gel but also affects the frequency dependent rheological properties.

\section{ACKNOWLEDGMENTS}

Majlinda Lako, Nicola Hunt, Ana Ferreira-Duarte, and Giorgia Montalbano are acknowledged for sample preparations. P. Duan and N. Kandemir are acknowledging Newcastle University to provide studentships. J. Chen is acknowledging funding from the Engineering and Physical Sciences Research Council (EP/K039083/1). 


\section{REFERENCES}

[1] S. V. Vlierberghe, P. Dubruel and E. Schacht, Biomacromolecules 12(5), 1387 (2011).

[2] K. Y. Lee and D. J. Mooney, Chem. Rev. 101(7), pp. 1869-1880 (2001).

[3] C. K. Kuo and P. X. Ma, Biomaterials 22(6), 511 (2001).

[4] A. Martinsen, G. Skjåk-Bræk and O. Smidsrød, Biotechnol. Bioeng. 33(1), 79 (1989).

[5] G. Klöck, A. Pfeffermann, C. Ryser, P. Gröhn, B. Kuttler, H. J. Hahn and U. Zimmermann, Biomaterials 18(10), 707 (1997).

[6] W. R. Gombotz and S. F. Wee, Adv. Drug Delivery Rev. 64, 194 (2012).

[7] J. A. Rowley, G. Madlambayan and D. J. Mooney, Biomaterials 20(1), 45 (1999).

[8] P. Fratzl (Ed.), Collagen: Structure and Mechanics, 1st ed (Springer, New York, 2008), p.113.

[9] S. J. Stagg, B. E. Pollot, C. R. Rathbone, A. Ong and T. Guda, Interpenetrating CollagenFibrin Hydrogels for Skeletal Muscle Regeneration, WWW Document, (http://2015.biomaterials.org/sites/default/files/abstracts/608.pdf), [Accessed 2204 2016].

[10] J. Chen, J. Irianto, S. Inamdar, P. Pravincumar, D. A. Lee, D. L. Bader and M. M. Knight, Biophys. J. 103(6), 1188 (2012).

[11] T. Funami, Y. Fang, S. Noda, S. Ishihara, M. Nakauma, K. I. Draget, K. Nishinari and G. O. Phillips, Food Hydrocoll. 23(7), 1746 (2009).

[12] D. M. Knapp, V. H. Barocas, A. G. Moon, K. Yoo, L. R. Petzold and R. T. Tranquillo, J. Rheol. 41(5), 971 (1998).

[13] C. B. e. a. da Cunha, Biomaterials 35(32), 8927 (2014). 